\title{
ON MODULAR GROUPS ISOMORPHIC WITH A GIVEN LINEAR
}

\section{GROUP*}

BY

\section{H. F. BLICHFELDT}

Theorem. Given a group $G$ of linear homogeneous substitutions in $n$ variables, transitive (irreducible) and of finite order. Then there exists an infinitude of prime numbers $p$ for each of which we can construct a simply isomorphic transitive group $G^{\prime}$ of linear homogeneous substitutions in $n$ variables, the elements of whose matrices are integers taken modulo $p$.

Let the operators of the abstract group $G^{\prime \prime}$ simply isomorphic with $G$ be $S_{i}^{\prime \prime}$, $i=1,2, \ldots, N$. Write down $N$ matrices in $n$ variables with undetermined coefficients

$$
S_{i}^{\prime}=\left\|a_{j k}^{i}\right\|,
$$

and form the $N^{2}$ products $S_{i}^{\prime} S_{j}^{\prime}$. Writing $S_{i}^{\prime} S_{j}^{\prime}=S_{k}^{\prime}$ whenever $S_{i}^{\prime \prime} S_{j}^{\prime \prime}=S_{k}^{\prime \prime}$, we obtain $n^{2} N^{2}$ equations in the elements $a_{j k}^{i}$. This system of equations shall be denoted by $A$. Any system of elements $a_{j k}^{i}$ satisfying $A$ will furnish a linear group $G_{1}$ isomorphic with $G^{\prime \prime}$. That this group may be transitive in $n$ variables we must, furthermore, have no equation of the form $\dagger$

$$
\sum_{j, k} b_{j k} a_{j k}^{i}=0
$$

$(i=1,2, \cdots, N)$

the coefficients $b_{j k}$ being independent of $i$. In other words, zero cannot be the value of every determinant of $\left(n^{2}\right)^{2}$ elements of the matrix of $n^{2}$ columns and $N$ rows, the $i$ th row of which is formed of the $n^{2}$ elements $a_{j k}^{i}$. We shall denote by $B^{\prime}$ the system of equations obtained by equating to zero all the determinants mentioned. Furthermore, in order that $G_{1}$ may not contain two transformations that are identical, we must exclude all possible sets of solutions of $A$ for which two rows of the matrix of $n^{2} N$ elements just mentioned are identical. This condition expressed in analytical form is as follows : the expression

\footnotetext{
* Presented to the Society (Chicago), April 14, 1906. Received for publication February 13, 1906.

† Burnside, Proceedings of the London Mathematical Society, vol. 3 (1905), p. 433; Frobenius, Sitzungsberichte der K. Preussischen Ak. der Wissenschaften, 1897, p. 1004.
} 


$$
P \equiv \prod_{i, i^{\prime}}\left\{\sum_{j, k} \lambda_{j k}\left(a_{j k}^{i}-a_{j k}^{i \prime}\right)\right\} \quad\left(\begin{array}{l}
j, k=1,2, \cdots, n^{2} ; \\
i, i^{\prime}=1,2, \cdots, N ; i \neq i^{\prime}
\end{array}\right)
$$

must not vanish for a set of $n^{2}$ arbitrary parameters $\lambda_{j k}$. We shall modify the system $B^{\prime}$ by multiplying each of its equations by $P$, and we shall denote the resulting set of equations by $B$.

Now, because the transitive group $G$ exists, the system $A$ can be solved, and solutions exist which will not satisfy all the equations of $B$. To solve $A$ we may, by a well known process, form a normal equation of the system, an algebraic equation whose coefficients are integers and which has no double roots. Let this equation be

$$
F=a_{e} x^{e}+a_{e-1} x^{e-1}+\cdots+a_{0}=0 .
$$

Denoting by $x$ any one of the roots of this equation, we can write every corresponding value of $\alpha_{j_{k}}^{i}$ as an integral function of $x$, the coefficients of which are definitely given rational numbers (the same for any root $x$ of (2) considered). Substituting in the system $B$ we have a series of equations in $x$ with rational coefficients, known functions of the parameters $\lambda_{j k}$, which equations could not all be satisfied for every root $x$ of (2). Hence $F=0$ has at least one root not found in one (say $C=0$ ) of the equations $B$. Let us suppose $F \equiv F_{1} F_{2}$, where $F_{1}=0$ has no root in common with $C=0$.* Then we can construct an identity of the form

$$
\alpha F_{1}+\beta C \equiv K_{1} \neq 0,
$$

where $\alpha, \beta$ and $\beta C$ are integral functions of $x$ whose coefficients, as well as $K_{1}$, are integral functions of the parameters $\lambda_{j k}$ with integral coefficients. To every root $x$ of $F_{1}=0$ will correspond a transitive group $G_{1}$ simply isomorphic with $G^{\prime \prime}$.

The question whether or not there exists a transitive linear group in $n$ variables simply isomorphic with $G^{\prime \prime}$ with coefficients modulo $p$ can now be solved. We start as above with the $N$ matrices

$$
S_{i}^{\prime}=\left\|a_{j k}^{i}\right\|
$$

and write down all the congruences $(\bmod p)$ following from the equations $S_{i}^{\prime} S_{j}^{\prime}=S_{k}^{\prime}$. The system $A$ above will merely be replaced by congruences, and instead of $F=F_{1} F_{2}=0$ we will have $F^{\prime}=F_{1} F_{2} \equiv 0(\bmod p)$. We remark that the coefficients of $F, F_{1}$ and $F_{2}$ are all known integers, although $p$ is, as yet, not known. The elements $a_{j k}^{i}$ are, as above, expressed as integral functions of a root $x$ of $F_{1} \equiv 0(\bmod p)$, the coefficients of which functions are known fractions. Let the least common multiple of all the denominators entering in these functions be denoted by $M$. We shall replace the parameters $\lambda_{j k}$ by such a system of integers that $K_{1}$ does not vanish. The resulting value of $K_{1}$ (an integer) will be denoted by $K$.

* We seek the highest common factor of $F$ and $C$, etc. The coefficients of $F_{1}$ and $F_{2}$ will be supposed to be integers. 
Suppose that $F_{1}=b_{m} x^{m}+\cdots+b_{0}$. We may assume that $b_{0} \neq 0$, as we may replace $x$ by $x+h$. Let us substitute for $x$ in $F_{1} \equiv 0(\bmod p)$ the quantity $M K b_{0} y$. We obtain

$$
b_{0}\left\{M K\left(c_{m} y^{m}+\cdots+c_{1} y\right)+1\right\} \equiv 0 \quad(\bmod p),
$$

the coefficients of the left-hand member being known integers evidently not all zero.

If we substitute any integer $y^{\prime}$ for $y$ such that

$$
M K\left(c_{m} y^{\prime m}+\cdots+c_{1} y^{\prime}\right)+1=L \neq 1 \text { or } 0,
$$

and choose for $p$ any prime factor $>1$ of $L$, we have a modulus $p$ fulfilling the conditions of the problem. For, $p$ is prime to $M K$, and $F_{1} \equiv 0(\bmod p)$ has a solution $x=M K b_{0} y^{\prime}$. Accordingly, the system of congruences $A$ is satisfied, but not the system $B$ (by virtue of the identity $\alpha F_{1}+\beta C \equiv K$ ). Because $A$ is satisfied, we have a modular group $H$ isomorphic with $G^{\prime \prime}$. If this group is intransitive modulo $p$, it may be transformed into a group of type

$$
\begin{array}{c|c}
H_{1} & 0 \\
\hline 0 & \frac{H_{2}}{2},
\end{array}
$$

from which it follows that the elements of $H$ satisfy at least one system of congruences corresponding to (1), from which again would follow the system $B$, and therefore also $C \equiv 0(\bmod p)$. Again, if $H$ were not simply isomorphic with $G$, the factor $P$ would vanish $(\bmod p)$, and therefore also every equation of $B$. But this is not the case, according to our procedure.

BERLIN,

January, 1906. 